\title{
Interstellar dust in the BOOMERanG maps
}

\author{
S. Masi ${ }^{1}$, P.A.R. Ade $^{2}$, J.J Bock ${ }^{3,4}$, A. Boscaleri ${ }^{5}$, B.P. Crill ${ }^{3}$, P. de Bernardis ${ }^{1}$, M. \\ Giacometti $^{1}$, E. Hivon ${ }^{3}$, V.V. Hristov ${ }^{3}$, A.E. Lange ${ }^{3}$, P.D. Mauskopf ${ }^{2}$, T. Montroy ${ }^{6}$, \\ C.B. Netterfield ${ }^{7}$, E. Pascale ${ }^{5}$, F. Piacentini ${ }^{1}$, S. Prunet ${ }^{8}$, J. Ruhl $^{6}$ \\ ${ }^{1}$ Dipartimento di Fisica, Universitá La Sapienza, Roma, P.le A. Moro, 2, 00185, Italy. ${ }^{2}$ Department of Physics \\ and Astronomy, Cardiff CF24 3YB, Wales, UK. ${ }^{3}$ California Institute of Technology, Pasadena, CA, USA. ${ }^{4}$ Jet \\ Propulsion Laboratory, Pasadena, CA, USA. ${ }^{5}$ IROE-CNR, Firenze, Italy. ${ }^{6}$ Dept. of Physics, Univ. of California, \\ Santa Barbara, CA, USA. ${ }^{7}$ Depts. of Physics and Astronomy, University of Toronto, Canada. ${ }^{8}$ C.I.T.A., \\ University of Toronto, Canada.
}

\begin{abstract}
Interstellar dust (ISD) emission is present in the mm-wave maps obtained by the BOOMERanG experiment at intermediate and high Galactic latitudes. We find that, while being sub-dominant at the lower frequencies $(90,150,240 \mathrm{GHz})$, thermal emission from ISD is dominant at $410 \mathrm{GHz}$, and is well correlated with the IRAS map at $100 \mu \mathrm{m}$. We find also that the angular power spectrum of ISD fluctuations at $410 \mathrm{GHz}$ is a power law, and its level is negligible with respect to the angular power spectrum of the Cosmic Microwave Background $(\mathrm{CMB})$ at 90 and $150 \mathrm{GHz}$.
\end{abstract}

\section{INTRODUCTION}

Interstellar dust, in the form of "cirrus" clouds, produce low level, patchy emission in the far-IR / mm-waves, even at high Galactic latitudes [1]. This has been recognized as a possible contaminant for sensitive CMB measurements at high frequencies (see e.g. [2], [3]) and also at low frequencies (see e.g. [4], [5]). Power spectra of this clumpy emission have been estimated from the IRAS survey at $3000 \mathrm{GHz}$ and from the DIRBE survey at $1250 \mathrm{GHz}$ [6] [7] [8] [9] [10]. All get $c_{\ell} \sim \ell^{-\beta}$ with $\beta \sim 2.5 \div 3$. In this paper we obtain a similar result for the data of the BOOMERanG map at 410 $\mathrm{GHz}$, and we use it to estimate the level of Galactic contamination in the BOOMERanG maps at 90, $150 \mathrm{and} 240 \mathrm{GHz}$. BOOMERanG [11], [12], [13], [14] has a dust monitor built-in, the $410 \mathrm{GHz}$ channel. This samples dust emission at a frequency much closer to the CMB than IRAS and DIRBE. In fact, the $410 \mathrm{GHz}$ map is dust dominated. We also detect dust emission at 90, 150 and $240 \mathrm{GHz}$. In the following we focus on the dust as a foreground, as in [15], and give a hint of new results relative to the temperature distribution of high latitude clouds.

\section{BOOMERANG DATA}

The BOOMERanG survey has been carried out at high and intermediate Galactic latitudes, in a southern sky region which contains the sky patch where interstellar dust emission is minimum. This region is approximately a rectangle in RA and dec, with $240^{\circ} \lesssim \ell \lesssim 290^{\circ}$ and $-50^{\circ} \lesssim b \lesssim-10^{\circ}$. In order to compare the BOOMERanG maps to other maps of the sky it is necessary to take into account the filtering procedure which has been applied to the time ordered data obtained during the scans. In fact, the signals from the BOOMERanG detectors have been high-pass filtered in the time domain to get rid of $1 / \mathrm{f}$ noise and drifts. A $16 \mathrm{mHz}$ hardware high pass was present in the amplification chain, and a $0.1 \mathrm{~Hz}$ high pass filter has been applied in the data reduction software. This means that the structures in the sky aligned to the scans and larger than $\sim 10^{\circ}$ have been effectively suppressed. Any comparison to other maps should be done by filtering them in the same way, and using the same map making procedure. We have applied such a procedure to the FDS maps [16] derived from the IRAS and DIRBE maps. The procedure consisted in convolving the FDS map with the BOOMERanG beam, then sample the convolved map in the same $57 \times 10^{6}$ directions observed by BOOMERanG, 
thus obtaining a fake time ordered dataset. This has been high pass filtered before applying the same map making procedures used for the BOOMERanG maps [17].

\section{THE 410 GHZ MAP}

The map at $410 \mathrm{GHz}$ and the FDS maps at the same frequency have been published in [15]. If one computes the difference of the two maps, only the cores of cold dust clouds, relatively brighter at $410 \mathrm{GHz}$ than at $3000 \mathrm{GHz}$, remain prominent in the difference map [15]. This means that the two components model of FDS [16] works remarkably well in general, but deviations are present in selected regions of the sky, pointing to a more complex dust distribution . We have computed the power spectrum of the brightness fluctuations at $410 \mathrm{GHz}$ in the three different regions of the $410 \mathrm{GHz}$ map. These sample three representative areas of the sky at low, intermediate and high Galactic latitudes. At low and intermediate latititudes the measured power spectra follow approximately power laws $c_{\ell} \sim \ell^{-2.3}$ and $c_{\ell} \sim \ell^{-3.1}$ respectively. At the high galactic latitudes the data are dominated by detector noise, and we can only give upper limits for the power spectrum of the sky. As expected because of the similarity of the maps, the spectra measured at $410 \mathrm{GHz}$ are consistent with corresponding spectra computed from the FDS maps [15].

\section{LONGER WAVELENGTHS}

At 90,150 and $240 \mathrm{GHz}$ the ISD emission is subdominant with respect to anisotropy of the CMB, at least at intermediate and high Galactic latitudes. We can search for a subdominant dust signal by correlating with the IRAS $3000 \mathrm{GHz}$ signal (or its FDS filtered extrapolation at the considered frequency). If we scatter plot the BOOMERanG signals (in the pixels of the map) against the comparison filtered FDS map, we expect that only ISD signals will produce a positive correlation. In fact, thermal emission from ISD has a broad spectrum peaking around $3000 \mathrm{GHz}$, but extending all the way to $90 \mathrm{GHz}$; on the contrary, the detector noise contributions to the signals in BOOMERanG and IRAS are uncorrelated, and the CMB anisotropy is present only in the bands measured by BOOMERanG. Its effect can only reduce the statistical significance of the positive correlation produced by ISD, but not change its estimate. We have produced scatter plots of this kind in several subregions of the sky observed by BOOMERanG. In fig.1,2,3 we plot some of these scatter plots.

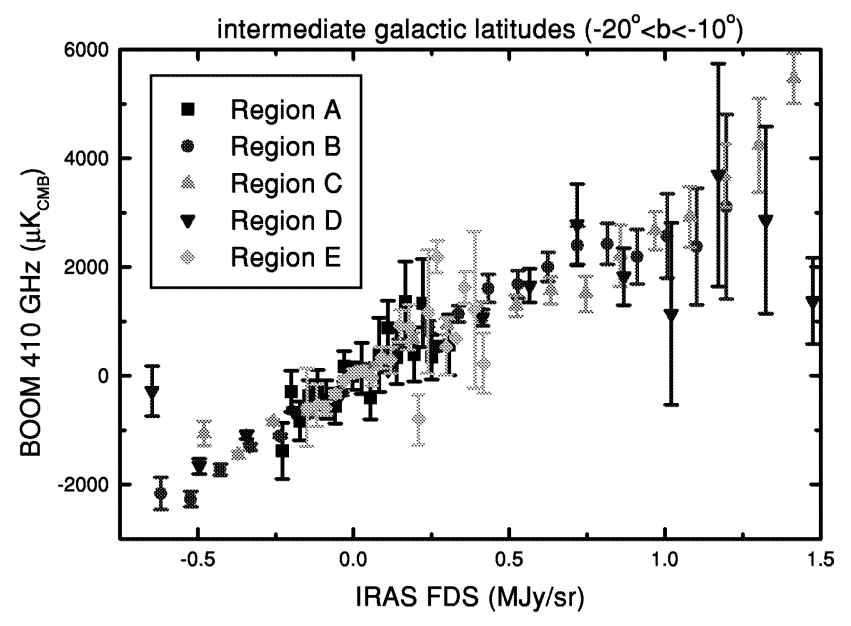

FIGURE 1. Scatter plot of the $410 \mathrm{GHz}$ data vs the IRAS data - intermediate Galactic latitudes.

The data have been averaged in equal IRAS brightness bins to help a visual evaluation of the correlation. The analysis, however, has been carried out on the original pixels. We use Pearson's linear correlation coefficient $R$ to estimate the statistical significance of the correlations, and the slope $S$ of the best fit line as an estimate of the average 


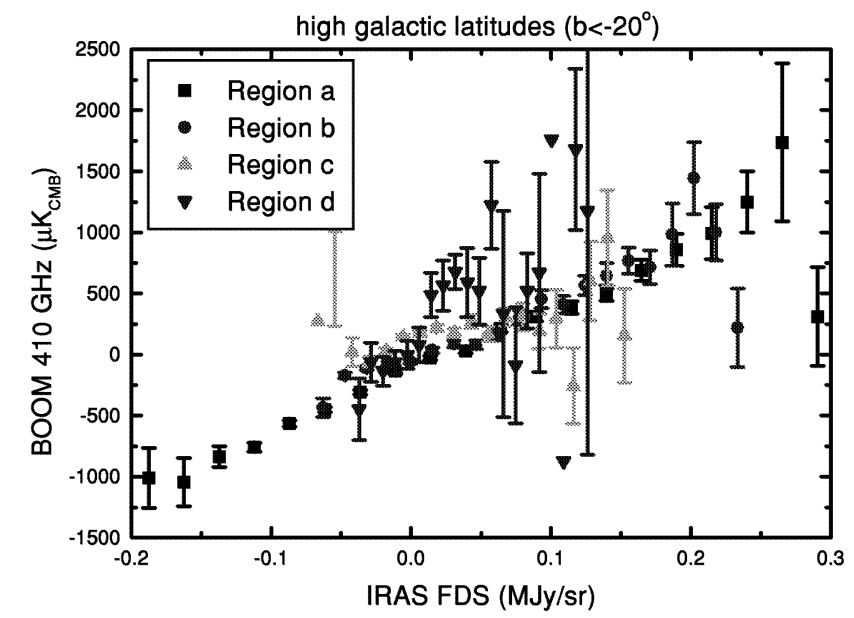

FIGURE 2. Scatter plot of the $410 \mathrm{GHz}$ data vs the IRAS data - high Galactic latitudes.

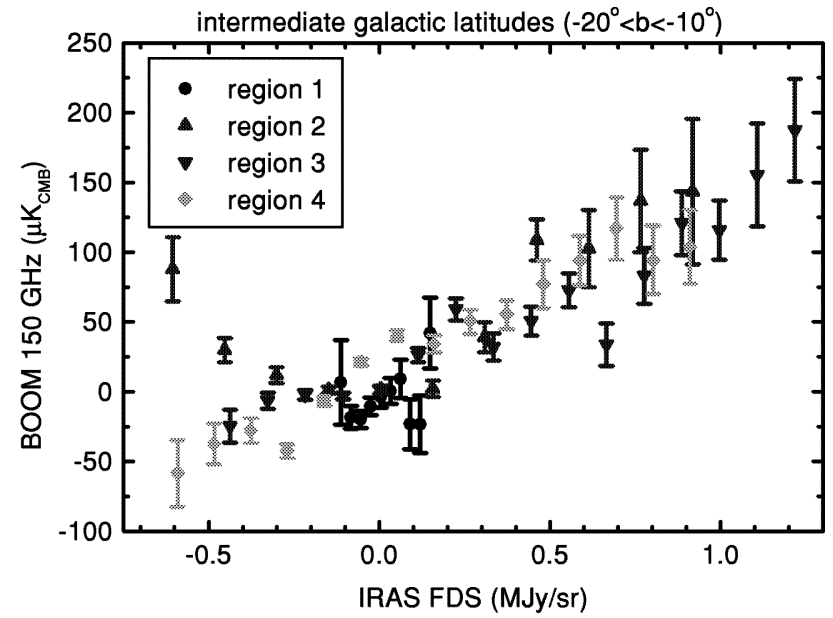

FIGURE 3. Scatter plot of the $150 \mathrm{GHz}$ data vs the IRAS data - intermediate Galactic latitudes.

ratio between ISD emission in the BOOMERanG maps and in the IRAS map. We divide the map in several regions, and for each region we compute the regression and the slope of the best fit. The average of the best fits is our best estimate of the general slope $S(\mathrm{v})$; the standard error on the average accounts for variations of dust properties and measurement errors. The results are visible in tables 1 and 2 . The correlation is statistically significant at intermediate Galactic latitudes in all channels, while at high Galactic latitudes is significant at 410 and $240 \mathrm{GHz}$.

Once they are converted in brightness units, the slopes $S(v)$ give the spectrum of dust brightness fluctuations normalized at $410 \mathrm{GHz}$. The resulting spectrum is consistent with a single temperature Rayleigh-Jeans thermal spectrum times an emissivity $\varepsilon=\varepsilon_{o} v^{\gamma}$, with $\gamma=(1.2 \pm 0.3)$ at low latitudes, and $\gamma=(2.3 \pm 1.0)$ at high latitudes. 
TABLE 1. Correlations at intermediate Galactic latitudes $\left(228437^{\prime}\right.$ pixels with $\left.-20^{\circ}<b<-10^{\circ}\right)$

\begin{tabular}{lcr}
\hline frequency $(\mathbf{G H z})$ & $R$ & $S\left(\mu K_{C M B} /(M J y / s r)\right)$ \\
\hline 90 & 0.032 & $58 \pm 49$ \\
150 & 0.085 & $93 \pm 23$ \\
240 & 0.156 & $254 \pm 46$ \\
410 & 0.298 & $3200 \pm 190$ \\
\hline
\end{tabular}

TABLE 2. Correlations at high Galactic latitudes $\left(689877^{\prime}\right.$ pixels with $b<-20^{\circ}$ )

\begin{tabular}{lrr}
\hline frequency $(\mathbf{G H z})$ & \multicolumn{1}{c}{$R$} & $S\left(\mu K_{C M B} /(M J y / s r)\right)$ \\
\hline 90 & -0.028 & $-20 \pm 110$ \\
150 & 0.003 & $46 \pm 29$ \\
240 & 0.041 & $258 \pm 52$ \\
410 & 0.138 & $4700 \pm 1500$ \\
\hline
\end{tabular}

\section{THE DUST AS A FOREGROUND}

Now we can combine the results obtained in the two previous paragraphs. The IRAS correlated dust fluctuations detected at $410 \mathrm{GHz}$ by BOOMERanG can be extrapolated to $240,150,90 \mathrm{GHz}$ using the measured slopes: $c_{\ell}(v)=$ $[S(v) / S(410)]^{2} c_{\ell}(410)$. In this way we find that the contamination from IRAS-correlated dust at $150 \mathrm{GHz}$ is two orders of magnitude below the measured power spectrum of the sky [15], thus confirming that the region of sky observed by BOOMERanG is dominated at $150 \mathrm{GHz}$ by CMB anisotropy. The contamination from the uncorrelated component depends on its spectrum. For reasonable spectra, it is smaller than the correlated part [15].

Our analysis shows that the FDS extrapolation works very well at intermediate latitudes, and within a factor 2 even at high Galactic latitudes. In the disk centered at $b=-27^{\circ}$ the rms fluctuation of the SFD IRAS map is $0.15 \mathrm{MJy} / \mathrm{sr}$ (extrapolated at $410 \mathrm{GHz}$ ). About $20 \%$ of the sky is as good or better than such disk, i.e. in about $20 \%$ of the sky the dust power spectrum is $<1 \%$ of the $\mathrm{CMB}$ power spectrum at $150 \mathrm{GHz}$.

Using multiband instruments, good foreground subtraction will be possible using components separation techniques, even in regions more contaminated than in our reference disk.

\section{COLD DUST CLOUDS}

We are studying several selected regions in the dust map of BOOMERanG. Particularily interesting are cold clouds, especially at high latitudes. In fig. 4 we show the same high latitude region in the IRAS map, in the BOOMERanG 410 $\mathrm{GHz}$ map, and in the map obtained by subtracting the $240 \mathrm{GHz}$ and the $150 \mathrm{GHz}$ maps. If the subtraction is carried out with the maps expressed in CMB temperature units, it removes efficiently the CMB anisotropy, leaving only the noise components and the dust, which in these units is much brighter at $240 \mathrm{GHz}$ than at $150 \mathrm{GHz}$. General agreement is evident from the comparison. A few clouds colder than the average are also evident in the comparison. We are currently investigating the physical nature of these clouds.

\section{CONCLUSIONS}

BOOMERanG has detected thermal emission from ISD cirrus at intermediate and high Galactic latitudes. The 410 $\mathrm{GHz}$ map is morphologically very similar to extrapolation of the IRAS $(3000 \mathrm{GHz})$ and DIRBE $(1250 \mathrm{GHz})$ maps. The angular power spectrum of the dust dominated $410 \mathrm{GHz}$ map is a power law $c_{\ell} \sim \ell^{-\beta}$ with $2 \lesssim \beta \lesssim 3$. We have detected a component correlated with the IRAS/DIRBE map in all the BOOMERanG bands at $-10^{\circ}>b>-20^{\circ}$, and in the 150, 240 and $410 \mathrm{GHz}$ bands at higher Galactic latitudes. This dust contamination is negligible with respect to the CMB anisotropy at high Galactic latitudes, accounting for less than $1 \%$ of the total angular power spectrum for multipoles $\ell>100$ at $v<180 \mathrm{GHz}$. 

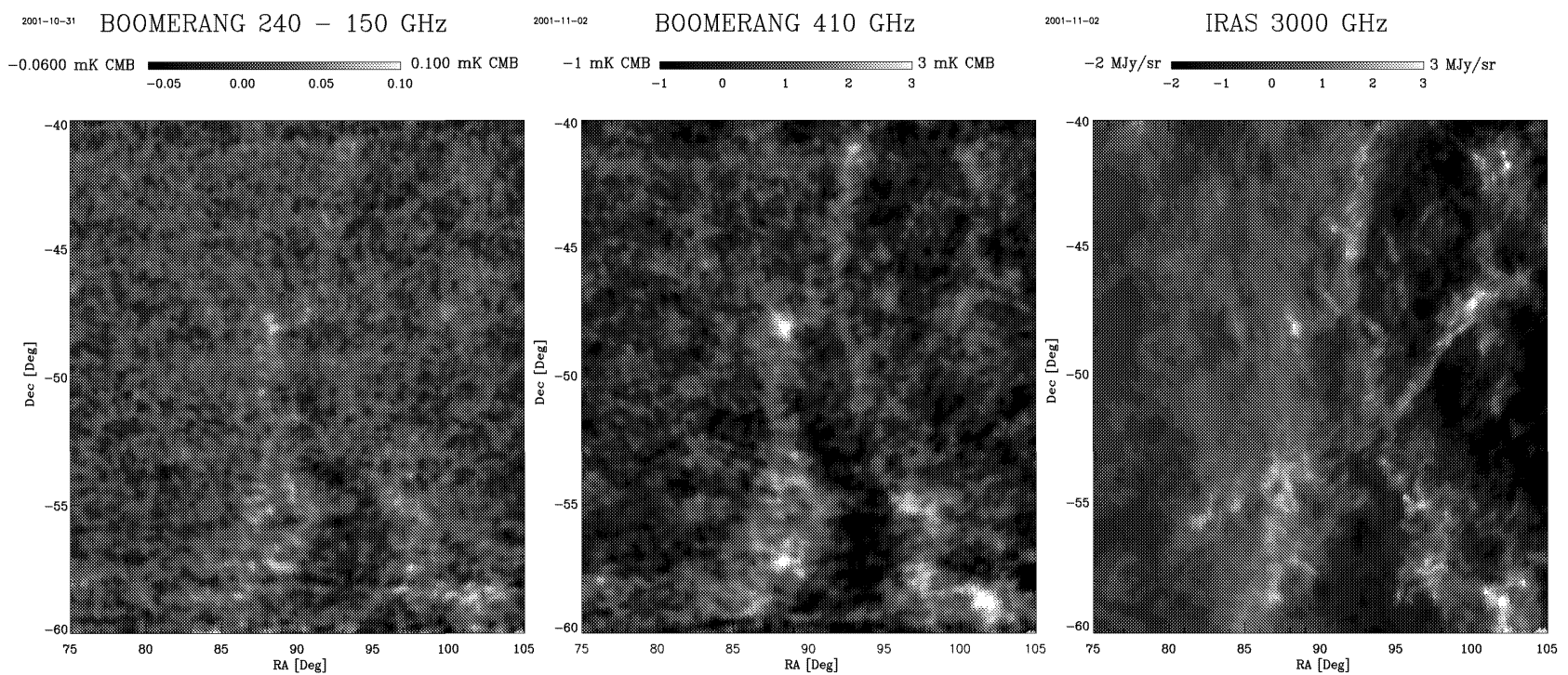

FIGURE 4. A high galactic latitude region with cirrus clouds as seen from BOOMERanG in the 240-150 GHz map (left panel), BOOMERanG (in the 410GHz map, center panel) and IRAS (in the $3000 \mathrm{GHz}$ map filtered in the same way as BOOMERanG right panel).

\section{ACKNOWLEDGMENTS}

This activity has been supported by the University of Rome La Sapienza, Programma Nazionale di Ricerche in Antartide and Agenzia Spaziale Italiana in Italy, by NASA and NSF in USA, by PPARC in UK and by Univ. of Toronto in Canada.

\section{REFERENCES}

1. Low F.J. et al. 1984, Ap.J. 278, L19.

2. Masi S. et al. 1995, Ap.J., 452, 253.

3. Masi S. et al. 1996, Ap.J., L47, 463.

4. Leitch E., et al. 1997, Ap.J., 486, L23.

5. de Oliveira-Costa 1997, Ap.J., 482, L17.

6. Gautier T.N. III, et al., 1992, A.J., 103, 4.

7. Low F.J. and Cutri R.M., 1994, Infrared Phys. Techn., 35, 291.

8. Guarini G. et al., 1995, Ap.J. 442, 23.

9. Wright E.L., 1998, Ap.J. 496, 1.

10. Schlegel D.J. et al. 1999, Ap.J. 500, 525.

11. Masi S., et al., in "3K cosmology", AIP Conf. Proc. 476, 237, (1999); astro-ph/9911520

12. de Bernardis, P., et al. 2000, Nature, 404, 955-959

13. Crill B. P., 2002, in preparation

14. Piacentini F., et al. 2001, "The BOOMERANG North America Instrument: a balloon-borne bolometric radiometer optimized for measurements of cosmic background radiation anisotropies from 0.3 to 4 degrees", astro-ph/0105148 (Ap.J. in press); see also his paper in this book.

15. Masi S., et al., 2001, Ap.J, 553, L93-L96, (astro-ph/0101539)

16. Finkbeiner D.P. et al. 1999, Ap.J., 524, 867.

17. Hivon E. et al., 2001, astro-ph/0105302 